\title{
Clinical and genetic relationships between the QTc interval and risk of a stroke among atrial fibrillation patients undergoing catheter ablation
}

Myunghee Hong ${ }^{\dagger}$, Kyeong-Hyeon Chun ${ }^{\dagger}{ }^{\prime}$ Inseok Hwang, Hee Tae Yu, Tae-Hoon Kim, Jae-Sun Uhm, Boyoung Joung, Moon-Hyoung Lee and Hui-Nam Pak *i)

\begin{abstract}
Background and objectives: A prolonged QTC interval is associated with an increased risk of a stroke or atrial fibrillation (AF). However, its direct causal relationship with AF associated a stroke has not been proven yet. To examine whether QTc interval is causally linked with risk of stroke in AF patients, we used the Mendelian randomization analysis.

Subjects and methods: Among 2742 patients (73.6\% male; $58.2 \pm 11.0$ years old; $69.5 \%$ with paroxysmal AF) who underwent AF catheter ablation, we analyzed 1766 patients who had preablation sinus rhythm electrocardiograms off antiarrhythmic drugs after excluding amiodarone users. Among them, 1213 subjects had genome-wide association study dataset analyzable for the Mendelian randomization. We explored the mechanistic relationships between QTc interval (ms) and the risk of a stroke by analyzing the Mendelian randomization (1213 subjects) after reviewing 35 genetic polymorphisms associated with the QTc in 31 European descent studies.
\end{abstract}

Results: Among the patients in the higher quartile with a higher QTc, $\mathrm{CHA}_{2} \mathrm{DS}_{2}$-VASc score $(p<0.001)$, and age $(p<0.001)$, the proportions of a prior stroke $(p<0.001)$, females, heart failure, and persistent AF were significantly higher than in those in the lower quartile. The QTc was independently associated with the $\mathrm{CHA}_{2} \mathrm{DS}_{2}-\mathrm{VASc}$ score $(\beta, 4.63 \mathrm{E}-5 ; 95 \%$ confidence interval, 3.57E-6-8.90E-5; $p=0.034)$ and ischemic strokes (odds ratio, 1.01; 95\% confidence interval, 1.00-1.01; $p=0.027$ ). However, there was no direct causal relationship between the QTc and $\mathrm{CHA}_{2} \mathrm{DS}_{2}$-VASc score or a prior stroke in either the one-sample or two-sample Mendelian randomizations.

Conclusion: The QTc was independently associated with the $\mathrm{CHA}_{2} \mathrm{DS}_{2}$-VASc score and strokes among the patients with AF who underwent catheter ablation, despite no genetically direct causal relationship.

Keywords: Atrial fibrillation, QT interval, Stroke, Mendelian randomization analysis

*Correspondence: hnpak@yuhs.ac

${ }^{\dagger}$ Myunghee Hong and Kyeong-Hyeon Chun have contributed equally to this work

Division of Cardiology, Department of Internal Medicine, Yonsei University Health System, 50-1 Yonseiro, Seodaemun-gu, Seoul 03722, Republic of Korea

\section{Introduction}

The QT interval represents the time duration between ventricular depolarization and repolarization, and a prolonged QT interval is known to be associated with cardiovascular death, sudden death, and all-cause death in the general population $[1,2]$. The QT interval reflects conduction abnormalities, electrolyte imbalances, or

(c) The Author(s) 2020. This article is licensed under a Creative Commons Attribution 4.0 International License, which permits use, sharing, adaptation, distribution and reproduction in any medium or format, as long as you give appropriate credit to the original author(s) and the source, provide a link to the Creative Commons licence, and indicate if changes were made. The images or other third party material in this article are included in the article's Creative Commons licence, unless indicated otherwise in a credit line to the material. If material is not included in the article's Creative Commons licence and your intended use is not permitted by statutory regulation or exceeds the permitted use, you will need to obtain permission directly from the copyright holder. To view a copy of this licence, visit http://creativeco mmons.org/licenses/by/4.0/. 
drug sensitivity related to ventricular repolarization. Atherosclerosis Risk in Communities (ARIC) studies have shown that a prolonged corrected QT (QTc) interval is associated with an increased risk of atrial fibrillation (AF) in the general population [3]. Hoshino et al. [4] reported that a prolonged QTc interval predicts poststroke paroxysmal AF. However, it is not clear whether the QTc is related to the risk or events of ischemic strokes in patients with AF.

$\mathrm{AF}$ is known to be a major underlying cause of cardioembolic strokes, and $20-25 \%$ of cases of ischemic strokes are associated with AF [5]. In patients with nonvalvular AF, heart failure, hypertension, old age, diabetes, vascular disease, and a female sex all play some role as risk factors for ischemic strokes, and the $\mathrm{CHA}_{2} \mathrm{DS}_{2}$-VASc score incorporating these factors has been used to assess the risk of a stroke [6]. Accordingly, we hypothesized that the QTc, a simple electrocardiographic (ECG) parameter, would exhibit a significant association with the $\mathrm{CHA}_{2} \mathrm{DS}_{2}$-VASc score and ischemic strokes in patients with nonvalvular AF. To test this hypothesis, we analyzed and compared the QTc indices to the clinical or prognostic factors and described the intracardiac electrophysiologic parameters in patients who had undergone AF catheter ablation (AFCA). We also attempted to document the mechanistic causal relationship between the QTc and risk or event of a stroke in patients with AF using the Mendelian randomization at the genetic level [7].

\section{Subjects and methods Study population}

The study protocol adhered to the Declaration of Helsinki and was approved by the Institutional Review Board of the Yonsei University Health System. All patients provided written informed consent for inclusion in the Yonsei AF Ablation Cohort Database (Clinical trials. gov NCT 02138695). The Institutional Review Board of Yonsei University approved this study protocol (IRB approval number 4-2010-0058). This study included 2742 consecutive patients with AF $(73.6 \%$ males; mean age $58.2 \pm 11.0$ years old) who underwent AFCA. After the exclusion of 647 patients who had taken amiodarone and 58 patients who did not have an analyzable sinus rhythm ECG, 1766 patients with preprocedural sinus rhythm ECGs without any antiarrhythmic effects were included in this study (Additional file 1: Fig. S1). Among the final patient population, $78.0 \%$ had paroxysmal AF (PAF) and $22.0 \%$ persistent AF (PeAF). There was a patient who experienced an aborted sudden cardiac arrest due to long QT syndrome. All antiarrhythmic drugs were discontinued for a period corresponding to at least five halflives. Anticoagulation therapy was maintained before the catheter ablation. A prior stroke event was defined as an ischemic stroke, and its diagnosis was based on a brain imaging study in each patient.

\section{Electrocardiographic measurement of the QTc interval} We analyzed the standard 12-lead ECGs in all patients (GE Healthcare, Marquette, MAC5500, Waukesha, WI). The paper speed was $25 \mathrm{~mm} / \mathrm{s}$, and the calibration was $10 \mathrm{~mm} / \mathrm{mV}$. The heart rate, PR interval, QRS, QTc, and P-axis were automatically measured by the ECG system. We used a single preprocedural ECG when sinus rhythm was maintained before the procedure in each patient without the effects of antiarrhythmic drugs for the analysis. The Bazett formula was used to calculate the QTc interval. As it is well known that there are sex differences in the QTc interval, a prolongation of the corrected QT interval was defined as $\geq 460 \mathrm{~ms}$ in women and $\geq 450 \mathrm{~ms}$ in men, as previously described $[8,9]$.

\section{Echocardiographic evaluation of the heart}

All patients underwent transthoracic echocardiography (Sonos 5500, Philips Medical System, Andover, MA, USA, or Vivid 7, GE Vingmed Ultrasound, Horten, Norway) prior to the AFCA. The chamber size, transmitral Doppler flow velocity, and ratio of the early diastolic peak mitral inflow velocity and early diastolic mitral annular velocity $(\mathrm{E} / \mathrm{Em})$ were acquired according to the American Society of Echocardiography guidelines [10]. Transesophageal echocardiography or intracardiac echocardiography was performed to exclude any intra-cardiac thrombi. The emptying velocity of the left atrial (LA) appendage was measured in all patients.

\section{Electroanatomical mapping and LA computed tomographic measurement}

Three-dimensional spiral computerized tomography (CT) scans (64-Channel, Light-Speed Volume CT, Philips, Brilliance 63, Amsterdam, Netherlands) were performed to visually define the pulmonary vein anatomy. The three-dimensional spiral CT images of the LA were analyzed on an imaging processing workstation (Aquarius, TeraRecon Inc., Foster city, CA, USA). A three-dimensional electroanatomical map (NavX, St. Jude Medical Inc., Minnetonka, MN, USA) was generated using a circular pulmonary vein mapping catheter (Lasso; Biosense-Webster Inc., Diamond Bar, CA, USA). NavX system-generated three-dimensional geometries of the LA and pulmonary vein were merged with the corresponding three-dimensional spiral CT images. We generated LA voltage maps by obtaining contact bipolar electrograms from over 500 points on the LA endocardium during atrial pacing at $500 \mathrm{~ms}$ and calculated the mean LA voltage as previously described $[11,12]$. 


\section{One-sample Mendelian randomization}

Among the 1766 included patients, the genome-wide association study (GWAS) results were available in 1213 patients who provided informed consent for a genetic study. Genomic DNA was extracted from peripheral blood samples using QuickGene DNA whole blood kits with QuickGene-Mini80 (KURABO, Osaka, Japan) and was analyzed using the Axiom Precision Medicine Research Array (PMRA, Thermo Fisher Scientific, Waltham, MA, USA). After quality control, 403402 genotyped SNPs were available for a Mendelian randomization (Additional file 1: Fig. S2). No genomic inflation was detected $(\lambda G C=1.002)$ after analyzing the QTc interval phenotype as a continuous variable. To identify a causal association between the QTc interval and strokes using the Mendelian randomization, we examined 35 independent SNPs of known QTc interval variants [13]. Of those SNPs, 29 SNPs were available, including two proxy SNPs $\left(r^{2}>0.8\right.$ in Asian sample from 1000 genomes Phase I).

\section{Two-sample Mendelian randomization in European populations}

We obtained publicly available summary statistics datasets from the MRBase database (http://app.mrbase.org/) to perform a two-sample Mendelian randomization. As exposure data, we selected known QT interval data (unit increase) for up to 100,000 individuals [13] from the NHGRI-EBI GWAS catalog (https://www.ebi.ac.uk/ gwas/). As outcome data, we used The International Stroke Genetics Consortium data, which consisted of 1859 cases with cardioembolic strokes and 19,326 controls [14].

For the two-sample Mendelian randomization analysis, we performed a clumping test to estimate the linkage disequilibrium between the instruments using data for 1000 genomes, because it is important to use independent genetic instruments for the exposure. After finishing the linkage disequilibrium clumping, we were able to use 24 independent SNPs related to the QT interval at genomewide significance levels $\left(p<5 \times 10^{-8}\right)$. We used summary statistics (beta coefficients and standard errors) for the 24 SNPs associated with the QTc interval levels from the QTc interval dataset (exposure). Also, we obtained the summary data (beta and standard errors) for the 24 SNPs from the cardioembolic stroke (outcome) dataset.

\section{Statistical analysis}

Normally distributed continuous variables are described as means \pm standard deviations. Intergroup comparisons for continuous variables were performed with the nonparametric Mann-Whitney $U$ test or paired $t$ test, as appropriate. The Chi-square test was used to assess the statistical significance of comparisons of categorical variables. Univariate and multivariate linear regression analyses were performed to identify the independent predictive value of QTc interval for the $\mathrm{CHA}_{2} \mathrm{DS}_{2}$-VASc score. Afterward, univariate and multivariate logistic regression analyses were performed to evaluate associations between the QTc interval and strokes. Variables that were associated with the QTc in the univariate analyses were included in the multivariate regression models. Missing values were excluded from the analyses, and a $p$ value $<0.05$ was considered statistically significant. To assess the associations of genetic variants with the QTc interval, we performed a linear regression. The $F$-statistics were used to determine the instrumental strength in the first stage. The causal effect between the QTc interval and strokes was quantified by a two-stage estimator approach. The Mendelian randomization analysis was performed with three models: (1) unadjusted model, (2) adjustment for the age and sex, and (3) adjustment for the age, heart failure, diabetes, hypertension, vascular disease, and a female sex. To use the weighted genetic risk score (wGRS) as an instrumental variable, we constructed a wGRS using four selected SNPs that were shown to be associated with the QTc interval. The wGRS was calculated as the sum of the risk alleles multiplied by the estimated $\beta$ coefficient (effect size) for each SNP. For the two-sample Mendelian randomization analysis, we used the MRBase database (http://app.mrbase.org/). To estimate the causal effect of the QTc interval (unit increase) on cardioembolic strokes, we performed the two-sample Mendelian randomization using inverse variance weighted (IVW), weighted median, and MR-Egger methods. All analyses were performed using IBM $^{\circledR}$ SPSS ${ }^{\circledR}$ Statistics 21 (IBM ${ }^{\circledR}$-Armonk, NY, USA), R version 3.5.1 (R Foundation for Statistical Computing, Vienna, Austria), and Stata/SE 15.1 (StataCorp, TX, USA) software.

\section{Results}

Clinical characteristics of AF patients with a prolonged QTC Table 1 summarizes the baseline clinical characteristics of the study individuals and compares the patients according to a quartile analysis of the QTc interval. Among the patients in the higher quartile of the QTc interval, the age $(p<0.001)$, proportion of females $(p<0.001)$, persistent $\operatorname{AF}(p<0.001)$, a prior stroke $(p<0.001)$, the $\mathrm{CHA}_{2} \mathrm{DS}_{2}$-VASc score $(p<0.001)$, and the presence of other associated cardiovascular comorbidities were significantly higher than those in the lower quartile of the QTc. In the ECG analysis, the patients with a QTc in the higher quartile had a higher ventricular rate $(p<0.001)$, longer PR interval $(p<0.001)$, and longer QRS duration $(p<0.001)$ than their counterparts (Table 1$)$. The LA 
Table 1 Baseline characteristics depending on the QTc interval

\begin{tabular}{|c|c|c|c|c|c|c|}
\hline & $\begin{array}{l}\text { All } \\
(N=1766)\end{array}$ & $\begin{array}{l}\text { Quartile } 1 \\
(N=450)\end{array}$ & $\begin{array}{l}\text { Quartile } 2 \\
(N=433)\end{array}$ & $\begin{array}{l}\text { Quartile } 3 \\
(N=441)\end{array}$ & $\begin{array}{l}\text { Quartile } 4 \\
(N=442)\end{array}$ & $p$ value \\
\hline QTc interval (ms) & $431.8 \pm 29.1$ & $397.0 \pm 13.3$ & $421.8 \pm 5.3$ & $439.6 \pm 5.4$ & $469.4 \pm 18.6$ & $<0.001$ \\
\hline Age (years) & $57.8 \pm 11.3$ & $54.9 \pm 11.6$ & $57.3 \pm 10.5$ & $58.7 \pm 10.8$ & $60.5 \pm 11.5$ & $<0.001$ \\
\hline Male $(n, \%)$ & $1260(71.3 \%)$ & $386(85.8 \%)$ & $329(76.0 \%)$ & $295(66.9 \%)$ & $250(56.6 \%)$ & $<0.001$ \\
\hline $\operatorname{PAF}(n, \%)$ & $1378(78.0 \%)$ & $388(86.2 \%)$ & $349(80.6 \%)$ & $337(76.4 \%)$ & $304(68.8 \%)$ & $<0.001$ \\
\hline $\mathrm{BMI}\left(\mathrm{kg} / \mathrm{m}^{2}\right)$ & $24.8 \pm 3.0$ & $24.7 \pm 3.1$ & $24.8 \pm 3.0$ & $24.8 \pm 2.8$ & $25.0 \pm 3.2$ & 0.762 \\
\hline $\mathrm{CHA}_{2} \mathrm{DS}_{2}$-VASc score & $1.6 \pm 1.5$ & $1.1 \pm 1.3$ & $1.4 \pm 1.3$ & $1.7 \pm 1.5$ & $2.2 \pm 1.7$ & $<0.001$ \\
\hline $\operatorname{CHF}(n, \%)$ & $93(5.3 \%)$ & $13(2.9 \%)$ & $9(2.1 \%)$ & $25(5.7 \%)$ & $46(10.4 \%)$ & $<0.001$ \\
\hline $\operatorname{HTN}(n, \%)$ & 791 (44.8\%) & $166(36.9 \%)$ & $182(42.0 \%)$ & $209(47.4 \%)$ & $234(52.9 \%)$ & $<0.001$ \\
\hline $\operatorname{DM}(n, \%)$ & $242(13.7 \%)$ & $50(11.1 \%)$ & $48(11.1 \%)$ & $71(16.1 \%)$ & $73(16.5 \%)$ & 0.017 \\
\hline Stroke $(n, \%)$ & $171(9.7 \%)$ & $29(6.4 \%)$ & $37(8.5 \%)$ & $42(9.5 \%)$ & $63(14.3 \%)$ & $<0.001$ \\
\hline $\operatorname{TIA}(n, \%)$ & $29(1.6 \%)$ & $9(2.0 \%)$ & $6(1.4 \%)$ & $10(2.3 \%)$ & $4(0.9 \%)$ & 0.378 \\
\hline Vascular disease $(n, \%)$ & 167 (9.5\%) & $38(8.4 \%)$ & $40(9.2 \%)$ & $36(8.2 \%)$ & $53(12.0 \%)$ & 0.192 \\
\hline Coronary artery disease $(n, \%)$ & $186(10.5 \%)$ & $36(8.0 \%)$ & $40(9.2 \%)$ & $41(9.3 \%)$ & $69(15.6 \%)$ & $<0.001$ \\
\hline eGFR $\left(\mathrm{ml} / \mathrm{min} / 1.73 \mathrm{~m}^{2}\right)$ & $81.8 \pm 15.4$ & $83.9 \pm 13.2$ & $83.1 \pm 13.6$ & $81.5 \pm 14.5$ & $78.5 \pm 19.2$ & $<0.001$ \\
\hline $\mathrm{HbA1c}(\%)$ & $6.4 \pm 0.9$ & $6.4 \pm 0.9$ & $6.3 \pm 0.9$ & $6.5 \pm 1.1$ & $6.4 \pm 0.8$ & 0.429 \\
\hline \multicolumn{7}{|l|}{ ECG parameters } \\
\hline Ventricular rate (bpm) & $61.3 \pm 9.8$ & $56.9 \pm 8.6$ & $60.6 \pm 8.8$ & $62.6 \pm 9.4$ & $65.2 \pm 10.5$ & $<0.001$ \\
\hline PR interval (ms) & $181.9 \pm 29.9$ & $176.6 \pm 26.0$ & $181.8 \pm 28.5$ & $181.9 \pm 31.5$ & $187.4 \pm 32.4$ & $<0.001$ \\
\hline QRS duration (ms) & $99.4 \pm 15.2$ & $97.0 \pm 11.4$ & $98.7 \pm 12.3$ & $98.2 \pm 13.8$ & $103.8 \pm 20.5$ & $<0.001$ \\
\hline \multicolumn{7}{|l|}{ Echocardiographic parameters } \\
\hline LA dimension (mm) & $40.3 \pm 5.9$ & $39.1 \pm 5.8$ & $40.2 \pm 5.9$ & $40.5 \pm 6.0$ & $41.5 \pm 5.6$ & $<0.001$ \\
\hline LA volume index $\left(\mathrm{ml} / \mathrm{m}^{2}\right)$ & $34.7 \pm 12.2$ & $31.6 \pm 11.2$ & $34.2 \pm 11.2$ & $35.3 \pm 12.4$ & $37.7 \pm 13.2$ & $<0.001$ \\
\hline LVEF (\%) & $64.2 \pm 7.1$ & $64.2 \pm 6.4$ & $65.3 \pm 6.5$ & $64.1 \pm 7.9$ & $63.4 \pm 7.4$ & 0.001 \\
\hline $\mathrm{E} / \mathrm{Em}$ & $9.9 \pm 3.9$ & $8.8 \pm 3.4$ & $9.7 \pm 3.5$ & $10.0 \pm 3.8$ & $11.3 \pm 4.6$ & $<0.001$ \\
\hline LVMI $\left(\mathrm{g} / \mathrm{m}^{2}\right)$ & $91.6 \pm 19.7$ & $89.8 \pm 17.6$ & $91.2 \pm 18.2$ & $91.9 \pm 20.8$ & $93.6 \pm 21.9$ & 0.061 \\
\hline \multicolumn{7}{|l|}{$3 D-C T$} \\
\hline LA volume (ml) & $141.2 \pm 47.3$ & $132.4 \pm 42.3$ & $138.6 \pm 48.2$ & $145.5 \pm 49.5$ & $148.6 \pm 47.3$ & $<0.001$ \\
\hline LA volume index $\left(\mathrm{ml} / \mathrm{m}^{2}\right)$ & $78.3 \pm 28.0$ & $71.5 \pm 24.6$ & $76.1 \pm 28.0$ & $81.0 \pm 29.0$ & $84.6 \pm 28.7$ & $<0.001$ \\
\hline NavX LA voltage (mV) & $1.3 \pm 0.6$ & $1.4 \pm 0.6$ & $1.3 \pm 0.6$ & $1.3 \pm 0.6$ & $1.2 \pm 0.6$ & $<0.001$ \\
\hline \multicolumn{7}{|l|}{ Medications } \\
\hline ACEI/ARB (\%) & $545(30.9 \%)$ & $113(25.1 \%)$ & $113(26.2 \%)$ & $140(31.8 \%)$ & $179(40.6 \%)$ & $<0.001$ \\
\hline Beta-blocker (\%) & $587(33.3 \%)$ & $145(32.2 \%)$ & $141(32.7 \%)$ & $150(34.1 \%)$ & $151(34.2 \%)$ & 0.896 \\
\hline Statin (\%) & $465(35.0 \%)$ & $104(23.1 \%)$ & $113(26.2 \%)$ & $117(26.6 \%)$ & $131(29.7 \%)$ & 0.172 \\
\hline
\end{tabular}

ACEI/ARB, angiotensin-converting enzyme inhibitors/angiotensin-receptor blockers; BMI, body mass index; $C H F$, congestive heart failure; DM, diabetes mellitus; $E C G$, electrocardiogram; E/Em, ratio of early diastolic transmitral flow velocity and mitral annular velocity; eGFR, estimated glomerular filtration rate; HbA1c, hemoglobin A1C; HTN, hypertension; LA, left atrial; LV EF, left ventricular ejection fraction; LVMI, left ventricular mass index; PAF, paroxysmal atrial fibrillation; TIA, transient ischemic attack

dimension $(p<0.001)$ and $\mathrm{E} / \mathrm{Em}(p<0.001)$ were higher and left ventricular ejection fraction $(p=0.001)$ and LA voltage $(p<0.001)$ significantly lower in the high quartile QTc group than low quartile group (Table 1). When we compared the patients with a prolonged QTc to those without according to the gender-specific criteria for the QTc, the results were consistent (Additional file 1: Table S1).

Additional file 1: Figure S3 shows representative examples of more advanced electroanatomical remodeling of the LA (greater LA volume index and lower LA endocardial voltage) in patients with a prolonged QTc. The QTc interval was significantly longer in the patients with heart failure, hypertension, an age $\geq 65$, a prior stroke, or a female sex (all $p<0.001$ ) than in those without (Fig. 1a). Figure 1b and c show the QTc distributions and its association with a prior stroke/transient ischemic attack (TIA) in patients with AF. 


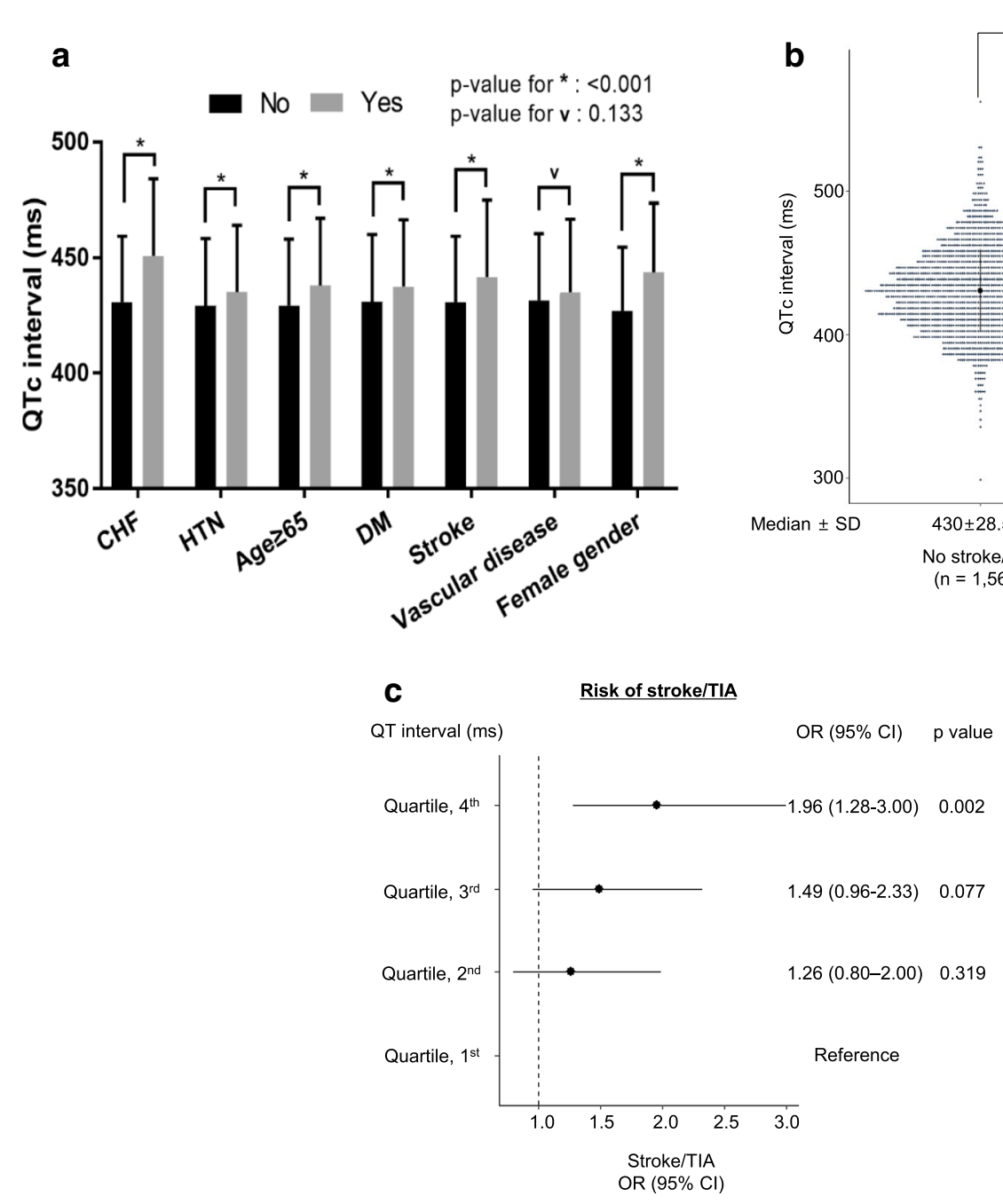

Fig. 1 a Comparison of the QTc intervals with respect to the clinical risk factors of the $\mathrm{CHA}_{2} \mathrm{DS}_{2}$-VASc score was expressed as bar graphs. Vascular disease includes prior a myocardial infarction, peripheral artery disease, and aortic plaque. b, c Observational association between the QTc interval and a stroke/TIA. b Association of the QTc interval according to that with or without a stroke/TIA. c Association of a stroke/TIA based on the quartile value of the QTc interval (ms)

The QTc is associated with the $\mathrm{CHA}_{2} \mathrm{DS}_{2}$-VASc score and a prior stroke

The QTc interval was significantly longer in the group with a $\mathrm{CHA}_{2} \mathrm{DS}_{2}$-VASc score $\geq 2 \quad(438.8 \pm 29.6 \mathrm{~ms})$ than in those with a score of 0 or $1(429.5 \pm 28.4 \mathrm{~ms}$; $p<0.001$; Mann-Whitney $U$ test), and the proportion of patients with a prolonged QTc was significantly higher in the patients with a $\mathrm{CHA}_{2} \mathrm{DS}_{2}$-VASc score $\geq 2(27.1 \%$, $209 / 770)$ than in those with a score of 0 or $1(17.6 \%$, 175/996, $p<0.001$; Chi-square test). In a multivariate linear regression analysis, the QTc $(\beta, 4.63 \mathrm{E}-5$; 95\% CI, $3.57 \mathrm{E}-6-8.90 \mathrm{E}-5 ; p=0.034)$ was independently associated with the $\mathrm{CHA}_{2} \mathrm{DS}_{2}$-VASc score (Table 2). In a multivariate logistic regression analysis, the QTc (OR,
1.007; 95\% CI, 1.001-1.013; $p=0.027)$ was independently associated with a prior stroke (Table 3 ).

\section{Evaluation of the causal effects by a one-sample Mendelian randomization}

Among the 35 SNPs known to be associated with the QTc, four SNPs, including RNF207 (rs846111), NOS1AP (rs12143842), SP3 (rs938291), and CNOT1 (rs246196), were associated with the QTc interval $(p<0.05$, Fig. 2a, left panel). However, they were not associated with a stroke or transient ischemic attack ( $p>0.05$, Fig. 2a, right panel). Next, we examined the four selected SNPs, including the highly associated SNP 
Table 2 Univariate and multivariate linear regression analyses for the parameters predicting $\mathrm{CHA}_{2} \mathrm{DS}_{2}$-VASc score

\begin{tabular}{|c|c|c|c|c|c|c|}
\hline & Univariate & & & Multivariat & & \\
\hline & $\beta$ & $95 \% \mathrm{Cl}$ & $p$ value & $\beta$ & $95 \% \mathrm{Cl}$ & $p$ value \\
\hline Age & 0.081 & $0.076-0.086$ & $<0.001$ & $-6.43 \mathrm{E}-5$ & $-2.21 \mathrm{E}-4-9.28 \mathrm{E}-5$ & 0.422 \\
\hline $\mathrm{CHF}$ & 1.442 & $1.133-1.752$ & $<0.001$ & 0.998 & $0.993-1.003$ & $<0.001$ \\
\hline HTN & 1.698 & $1.579-1.816$ & $<0.001$ & 1.001 & $0.998-1.003$ & $<0.001$ \\
\hline DM & 1.942 & $1.757-2.126$ & $<0.001$ & 0.998 & $0.995-1.002$ & $<0.001$ \\
\hline Age $\geq 75$ years & 1.519 & $1.382-1.657$ & $<0.001$ & 1.000 & $0.997-1.003$ & $<0.001$ \\
\hline Age $65-74$ years & 1.546 & $1.398-1.695$ & $<0.001$ & 1.003 & $0.999-1.006$ & $<0.001$ \\
\hline Prior stroke/TIA & 1.354 & $1.262-1.447$ & $<0.001$ & 0.999 & $0.998-1.001$ & $<0.001$ \\
\hline Vascular disease & 1.988 & $1.764-2.211$ & $<0.001$ & 1.006 & $1.002-1.010$ & $<0.001$ \\
\hline Female sex & 1.289 & $1.144-1.433$ & $<0.001$ & 0.999 & $0.996-1.001$ & $<0.001$ \\
\hline PAF & -0.253 & -0.424 to -0.083 & 0.004 & -0.002 & $-0.005-0.001$ & 0.136 \\
\hline BMI & $1.94 \mathrm{E}-4$ & $-0.001-0.002$ & 0.803 & & & \\
\hline LA dimension & 0.049 & $0.043-0.055$ & $<0.001$ & $3.22 \mathrm{E}-5$ & $-1.79 \mathrm{E}-4-2.44 \mathrm{E}-4$ & 0.765 \\
\hline PR interval & 0.004 & $0.001-0.006$ & 0.002 & $-1.13 \mathrm{E}-5$ & $-5.08 \mathrm{E}-5-2.83 \mathrm{E}-5$ & 0.576 \\
\hline QRS & -0.009 & -0.013 to -0.004 & $<0.001$ & $-8.84 \mathrm{E}-6$ & $-8.91 \mathrm{E}-5-7.14 \mathrm{E}-5$ & 0.829 \\
\hline QTc interval & 0.014 & $0.012-0.015$ & $<0.001$ & $4.63 E-5$ & $3.57 \mathrm{E}-6-8.90 \mathrm{E}-5$ & 0.034 \\
\hline
\end{tabular}

$\beta$, regression coefficient; $\mathrm{BMI}$, body mass index; $\mathrm{CHF}$, congestive heart failure; $\mathrm{Cl}$, confidence interval; $\mathrm{DM}$, diabetes mellitus; $\mathrm{HTN}$, hypertension; $\mathrm{LA}$, left atrial; PAF, paroxysmal atrial fibrillation; TIA, transient ischemic attack

Table 3 Multivariate logistic regression analyses for the noninvasive parameters associated with a stroke

\begin{tabular}{|c|c|c|c|c|c|c|}
\hline & \multicolumn{3}{|c|}{ Univariate } & \multicolumn{3}{|c|}{ Multivariate } \\
\hline & OR & $95 \% \mathrm{Cl}$ & $p$ value & OR & $95 \% \mathrm{Cl}$ & $p$ value \\
\hline QTc interval & 1.012 & $1.007-1.018$ & $<0.001$ & 1.007 & $1.001-1.013$ & 0.027 \\
\hline Heart failure & 1.411 & $0.753-2.643$ & 0.283 & & & \\
\hline Hypertension & 1.573 & $1.145-2.160$ & 0.005 & 0.775 & $0.533-1.128$ & 0.775 \\
\hline Age & 1.057 & $1.040-1.074$ & $<0.001$ & 1.049 & $1.029-1.069$ & $<0.001$ \\
\hline Diabetes & 3.020 & $2.102-4.338$ & $<0.001$ & 2.117 & $1.410-3.177$ & $<0.001$ \\
\hline Vascular disease & 2.156 & $1.394-3.334$ & 0.001 & 1.183 & $0.721-1.942$ & 0.506 \\
\hline Female sex & 1.326 & $0.949-1.854$ & 0.098 & 0.898 & $0.601-1.342$ & 0.601 \\
\hline LA dimension & 1.054 & $1.027-1.083$ & $<0.001$ & 1.033 & $1.000-1.067$ & 0.050 \\
\hline $\mathrm{E} / \mathrm{Em}$ & 1.109 & $1.072-1.148$ & $<0.001$ & 1.046 & $1.002-1.091$ & 0.039 \\
\hline
\end{tabular}

$\mathrm{Cl}$, confidence interval; $\mathrm{E} / \mathrm{Em}$, ratio of the early diastolic transmitral flow velocity and mitral annular velocity; $\mathrm{OR}$, odds ratio

rs12143842 located near NOS1AP, and their wGRS by a two-stage estimator method. The F-statistics of the top SNP rs12143842 ( $F$-statistics $=22.19)$ and wGRS $(F$-statistics $=25.17)$ in the first-stage regression were indicated to have enough strength as to be used as instrumental variables after adjusting for the age, heart failure, diabetes, hypertension, vascular disease, and a female sex (Additional file 1: Table S2). However, in the two-stage regression, the results of the Mendelian randomization analysis were not significant for any instrumental variable (OR, 0.99; 95\% CI, 0.92-1.06; $p=0.729$ in rs12143842; OR, 1.00; 95\% CI, 0.96-1.04; $p=0.988$ in wGRS, Fig. 2b).

Two-sample Mendelian randomization in the European population

To obtain supportive evidence of an independent association between the QT interval and cardioembolic strokes in European populations, we performed a twosample Mendelian randomization using the MRBase database. The IVW method did not reveal any evidence of a causal relationship (OR estimate, 0.996; 95\% CI, 0.98-1.01; $p=0.555)$ between the QT interval and 


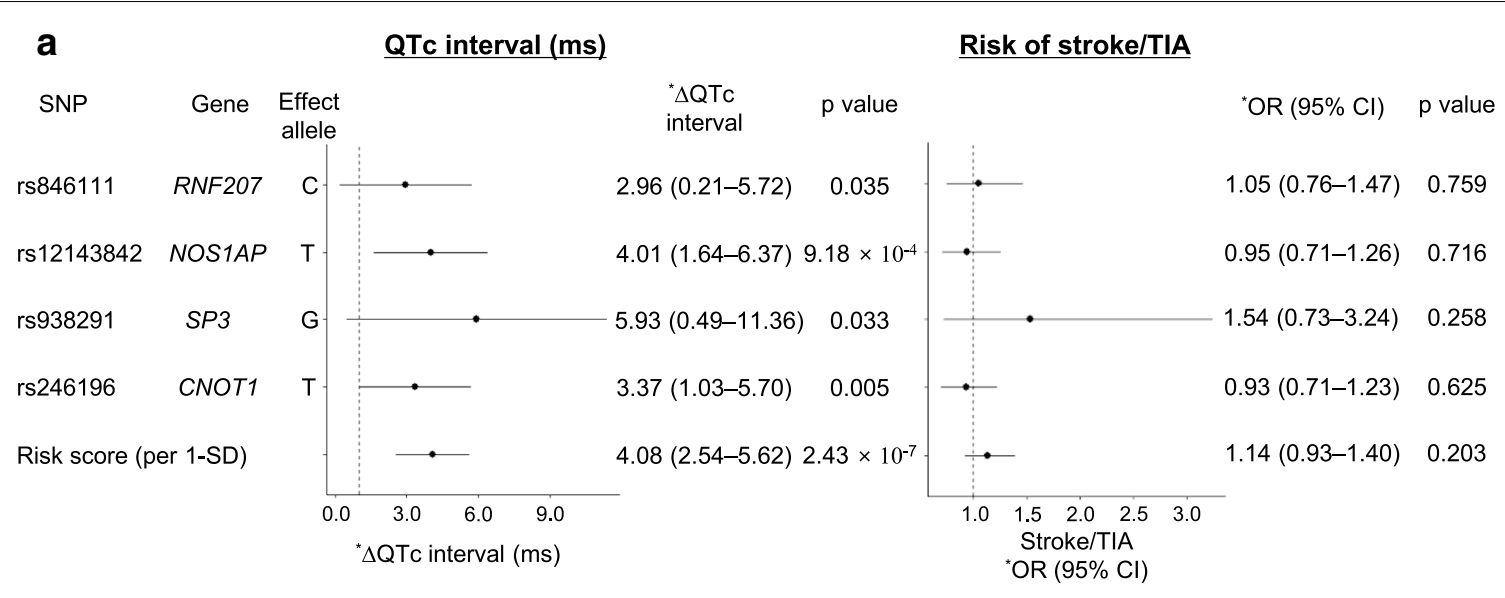

${ }^{*}$ Age and sex adjusted

b

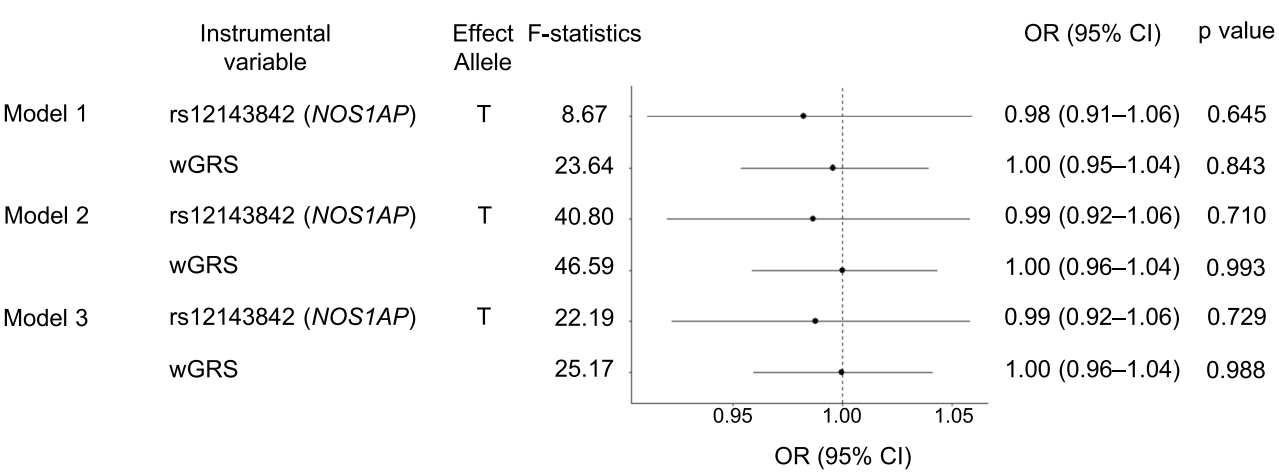

Model1: unadjusted; Model 2: Adjusted by age and sex; Model 3: Adjusted by age, heart failure, diabetes, hypertension, vascular disease, female.

Fig. 2 The causal association of the QTc interval on a stroke/TIA. a Association of four genetic variants and the weighted genetic risk score (wGRS) with the QTc interval (exposure) and a stroke/TIA (outcome). b The results of the one-sample Mendelian randomization in the top SNP rs 12143842 (NOS1AP at 1q23.3) and wGRS for the 4 SNPs

strokes using 24 independent instrumental variables (Additional file 1: Table S3). The MR-Egger regression method also did not support a causal relationship (OR estimate, 0.996; 95\% CI, 0.97-1.02; $p=0.726$ ) without bias from directional pleiotropy (intercept $=-0.001$; $\mathrm{SE}=0.013$; directionality $p=0.929)$, nor did the weighted median method (OR estimate, 0.996; 95\% CI, $0.98-1.01 ; p=0.694$ ) (Table 4, Additional file 1: Fig. S4). To test the heterogeneity between the instrumental variable estimates for individual SNPs, we performed a Cochran's $Q$ test. The results of the heterogeneity test for IVW and MR-Egger were a $p>0.05$ (Table 4).

Table 4 Two-sample Mendelian randomization estimates of the causal effect of QT interval on cardioembolic stroke

\begin{tabular}{|c|c|c|c|c|c|c|}
\hline Method & Number of SNP & OR estimate & $95 \% \mathrm{Cl}$ & $p$ value & $\begin{array}{l}\text { Cochran } Q \\
\text { statistic }\end{array}$ & $\begin{array}{l}\text { Heterogeneity } \\
p \text { value }\end{array}$ \\
\hline MR-Egger & 24 & 0.996 & $0.97-1.02$ & 0.726 & 13.91 & 0.905 \\
\hline Inverse variance weighted & 24 & 0.996 & $0.98-1.01$ & 0.555 & 13.92 & 0.929 \\
\hline Weighted median & 24 & 0.996 & $0.98-1.01$ & 0.694 & NA & NA \\
\hline
\end{tabular}

$\mathrm{Cl}$, confidence interval; MR, Mendelian randomization; NA, not available; OR, odds ratio; SNP, single nucleotide polymorphism 


\section{Discussion}

In this retrospective analyses of single-center prospective registry study, we systematically analyzed the clinical and genetic characteristics of the patients with AF according to the QTc. In this study, we found that a prolonged QTc was independently associated with the $\mathrm{CHA}_{2} \mathrm{DS}_{2}$-VASc score and prior stroke events. However, there was no genetic causal relationship between the QTc and AFassociated ischemic strokes in either the one-sample or two-sample Mendelian randomization. To the best of our knowledge, this might be the first study to describe an indirect relationship between the QTc interval and AFassociated ischemic strokes through clinical and genetic analyses.

\section{A prolonged QTc as a risk factor for a stroke in AF}

The QTc is dependent on the ventricular depolarization and repolarization times. Research has established that a QTc prolongation is a predictor of a stroke in the general population $[8,15]$. The QTc interval is also associated with diabetes [16], obesity [17], and subclinical atherosclerosis [18] and can be used to predict atherosclerotic vascular events, including strokes [8]. Although there are several reports on the associations between a prolonged QTc and the prevalence of AF or poststroke subclinical paroxysmal AF $[3,4,19]$, its direct relationship in patients with AF and a prior stroke has never been reported. In this study, we showed that the QTc was independently associated with both the $\mathrm{CHA}_{2} \mathrm{DS}_{2}$-VASc score and a prior stroke among patients who underwent AFCA. However, we still could not exclude the risk of a potential noncardioembolic stroke, because the risks of both cardioembolic and noncardioembolic strokes increase simultaneously as the $\mathrm{CHA}_{2} \mathrm{DS}_{2}$-VASc score increases [20].

\section{Mechanistic linkage between a prolonged QTC and higher AF burden}

The QTc interval is known to be affected by cardiovascular conditions, such as aging, high blood pressure, metabolic disorders, ischemic heart disease, or cerebrovascular disease [21, 22]. There are several potential explanations for the relationship between a prolonged QTc and the AF burden. First, a prolongation of the QT interval is known to be present in heart failure and ventricular hypertrophy [23]. Under the condition of heart failure, a chronic volume or pressure overload increases the LA pressure, which facilitates AF [24]. Second, a higher AF burden may lead to deterioration of the ventricular function and QTc prolongation [25]. In addition, neuro-hormonal effects in heart failure also can affect the QT interval [26]. Third, a QTc prolongation itself can mediate susceptibility to AF as a purely electrical problem, as suggested by "atrial torsades de pointes [19]." Genome-wide linkage studies of one family have identified a genetic locus associated with neonatal AF with variable cardiomyopathy and sudden death [27]. These findings indicate that a prolonged QTc is related to heart failure and $\mathrm{AF}$, both of which are major risk factors for cardioembolic and noncardioembolic strokes.

\section{Mendelian randomization}

Although we found statistically significant associations for the QTc prolongation with a prior stroke and $\mathrm{CHA}_{2} \mathrm{DS}_{2}$-VASc score in patients with $\mathrm{AF}$, there was no genetic evidence for the causal effect between the QTc and strokes upon a Mendelian randomization. This method uses measured variations in the genes of known function to examine the causal effect of a modifiable exposure on a disease in observational studies [28, 29]. These findings suggest that the QTc interval is closely related to strokes in patients with AF, but is affected by multiple confounding factors without a direct causal relationship. Although our sample size was relatively small, it had statistically enough instrumental strength in the first stage to detect a causal association by the Mendelian randomization. Generally, the instrumental variables with F-statistics less than 10 are often labeled as weak instruments [7].

In addition, to provide supportive evidence of these findings, we used a two-sample Mendelian randomization analysis between the QTc interval and cardioembolic strokes using previously published summary data in European populations [13, 14]. We found consistently negative findings by the two-sample Mendelian randomization in the IVW, weighted median, and MR-Egger methods.

\section{Limitations}

This study was an observational study from a cohort registry that included a highly selective group of patients who were referred for AFCA. Therefore, the results of this study cannot be generalized to the overall AF population. We excluded 305 patients who did not show sinus rhythm ECG after stopping antiarrhythmic drugs or amiodarone users. Also, we only analyzed a single ECG before each procedure. It would be most ideal if prospective studies are able to show whether the incidence of actual stroke increases in AF patients with a prolonged QTc. In this point of view, multiple ECGs or Holter monitoring might be of help to compensate for variations and to obtain more significant analyses. We conducted this study based on the previously published summary data in European descent those cannot be generalized to the overall AF genetics. 


\section{Conclusion}

In this study, a prolonged QTc was independently associated with the $\mathrm{CHA}_{2} \mathrm{DS}_{2}$-VASc score and prior stroke events. However, there was no genetic causal relationship between the QTc and AF-associated ischemic strokes in either the one-sample or two-sample Mendelian randomization.

\section{Supplementary information}

Supplementary information accompanies this paper at https://doi. org/10.1186/s42444-020-00017-5.

Additional file 1. Table S1. Baseline characteristics with a comparison between the patients with a normal QTc and prolonged QTc. Table S2. One-sample Mendelian randomization analysis: the causal association of QTc interval and stroke using genetic variants. Table S3. Association of 24 single instrumental variables for QT interval and cardioembolic stroke in European populations. Fig. S1. Study flowchart of the patient enrolment. Fig. S2. A Manhattan plot for the QTC interval (ms) phenotype in 1,213 patients with atrial fibrillation. Fig. S3. Patients with a prolonged QTC interval had a tendency towards advanced electroanatomical remodeling of the left atrium and a prior stroke. Fig. S4. Two-sample Mendelian randomization analysis.

\section{Abbreviations}

AF: atrial fibrillation; AFCA: atrial fibrillation catheter ablation; ARIC: Atherosclerosis Risk in Communities; $\beta$ : beta coefficient; CT: computerized tomography; ECG: electrocardiogram; E/Em: ratio of the early diastolic peak mitral inflow velocity and early diastolic mitral annular velocity; eGFR: estimated glomerular filtration rate; GWAS: genome-wide association study; IVW: inverse variance weighted; LA: left atrial; MR: Mendelian randomization; PAF: paroxysmal atrial fibrillation; PeAF: persistent atrial fibrillation; QTc: corrected QT interval; SE: standard error; TIA: transient ischemic attack; WGRS: weighted genetic risk score.

\section{Acknowledgements}

We would like to thank Mr. John Martin for his linguistic assistance.

\section{Authors' contributions}

HNP and MH obtained financial support and conceived the study. HNP and KHC designed the study, assessed clinical phenotyping, and performed data analyses. HNP, MH, and IH supervised genotyping, data analysis, and interpretation. MH and KHC drafted the manuscript. HNP and HTY enrolled the subjects and acquired informed consents. HTY, THK, J-SU, BJ, MHL, and HNP contributed to conceptualization of the study and conducting the study procedures. HNP and $\mathrm{MH}$ substantially contributed to revising the article and researching data. All authors read and approved the final manuscript.

\section{Funding}

This work was supported by Grants [HI18C0070 to P H-N] and [HI19C0114 to $\mathrm{P} \mathrm{H-N}$ ] from the Ministry of Health and Welfare and Grants [NRF$2017 R 1 A 2 B 4003983$ to P H-N and NRF-2019R1I1A1A01041440 to H.M] from the Basic Science Research Program run by the National Research Foundation of Korea.

\section{Competing interests}

The authors declare that they have no competing interests.

\section{Availability of data and materials \\ Not applicable.}

\section{Consent for publication}

Not applicable.

\section{Ethics approval and consent to participate}

This study was approved by the Institutional Review Board of the Yonsei University Health System. All patients provided written informed consent for inclusion in the Yonsei AF Ablation Cohort Database (Clinical trials.gov NCT 02138695).

Received: 7 January 2020 Accepted: 28 May 2020

Published online: 18 June 2020

\section{References}

1. Nielsen JB, Graff C, Rasmussen PV, Pietersen A, Lind B, Olesen MS, Struijk JJ, Haunso S, Svendsen JH, Kober L, Gerds TA, Holst AG. Risk prediction of cardiovascular death based on the QTc interval: evaluating age and gender differences in a large primary care population. Eur Heart J. 2014;35(20):1335-44.

2. Opthof T, Coronel R, Janse MJ. Is there a significant transmural gradient in repolarization time in the intact heart? Repolarization gradients in the intact heart. Circ Arrhythm Electrophysiol. 2009;2(1):89-96.

3. Mandyam MC, Soliman EZ, Alonso A, Dewland TA, Heckbert SR, Vittinghoff E, Cummings SR, Ellinor PT, Chaitman BR, Stocke K, Applegate WB, Arking DE, Butler J, Loehr LR, Magnani JW, Murphy RA, Satterfield S, Newman $A B$, Marcus $G M$. The QT interval and risk of incident atrial fibrillation. Heart Rhythm. 2013;10(10):1562-8.

4. Hoshino T, Nagao T, Shiga T, Maruyama K, Toi S, Mizuno S, Ishizuka K, Shimizu S, Uchiyama S, Kitagawa K. Prolonged QTc interval predicts poststroke paroxysmal atrial fibrillation. Stroke. 2015;46(1):71-6.

5. Mandzia JL, Hill MD. Acute stroke management in patients with known or suspected atrial fibrillation. Can J Cardiol. 2013;29(7 Suppl):S45-53.

6. Chen JY, Zhang AD, Lu HY, Guo J, Wang FF, Li ZC. CHADS2 versus CHA2DS2-VASc score in assessing the stroke and thromboembolism risk stratification in patients with atrial fibrillation: a systematic review and meta-analysis. J Geriatr Cardiol. 2013;10(3):258-66.

7. Burgess S, Small DS, Thompson SG. A review of instrumental variable estimators for Mendelian randomization. Stat Methods Med Res. 2017;26(5):2333-55.

8. Soliman EZ, Howard G, Cushman M, Kissela B, Kleindorfer D, Le A, Judd S, McClure LA, Howard VJ. Prolongation of QTC and risk of stroke: the REGARDS (REasons for Geographic and Racial Differences in Stroke) study. J Am Coll Cardiol. 2012;59(16):1460-7.

9. Rautaharju PM, Surawicz B, Gettes LS, Bailey JJ, Childers R, Deal BJ, Gorgels A, Hancock EW, Josephson M, Kligfield P, Kors JA, Macfarlane P, Mason JW, Mirvis DM, Okin P, Pahlm O, van Herpen G, Wagner GS, Wellens H, American Heart Association E, American College of Cardiology F. AHA/ ACCF/HRS recommendations for the standardization and interpretation of the electrocardiogram: part IV: the ST segment, T and U waves, and the QT interval: a scientific statement from the American Heart Association Electrocardiography and Arrhythmias Committee, Council on Clinical Cardiology; the American College of Cardiology Foundation; and the Heart Rhythm Society. Endorsed by the International Society for Computerized Electrocardiology. J Am Coll Cardiol. 2009;53(11):982-91.

10. Nagueh SF, Appleton CP, Gillebert TC, Marino PN, Oh JK, Smiseth OA, Waggoner AD, Flachskampf FA, Pellikka PA, Evangelista A. Recommendations for the evaluation of left ventricular diastolic function by echocardiography. J Am Soc Echocardiogr. 2009;22(2):107-33.

11. Lin YJ, Tai CT, Kao T, Chang SL, Wongcharoen W, Lo LW, Tuan TC, Udyavar AR, Chen YJ, Higa S, Ueng KC, Chen SA. Consistency of complex fractionated atrial electrograms during atrial fibrillation. Heart Rhythm. 2008;5(3):406-12.

12. Park JH, Pak HN, Kim SK, Jang JK, Choi Jl, Lim HE, Hwang C, Kim YH. Electrophysiologic characteristics of complex fractionated atrial electrograms in patients with atrial fibrillation. J Cardiovasc Electrophysiol. 2009;20(3):266-72.

13. Arking DE, Pulit SL, Crotti L, van der Harst P, Munroe PB, Koopmann TT, Sotoodehnia N, Rossin EJ, Morley M, Wang X, et al. Genetic association study of QT interval highlights role for calcium signaling pathways in myocardial repolarization. Nat Genet. 2014;46(8):826-36.

14. Malik R, Traylor M, Pulit SL, Bevan S, Hopewell JC, Holliday EG, Zhao W, Abrantes P, Amouyel P, Attia JR, Battey TW, Berger K, Boncoraglio GB, Chauhan G, Cheng YC, Chen WM, Clarke R, Cotlarciuc I, Debette 
S, Falcone GJ, Ferro JM, Gamble DM, llinca A, Kittner SJ, Kourkoulis CE, Lemmens R, Levi CR, Lichtner P, Lindgren A, Liu J, Meschia JF, Mitchell BD, Oliveira SA, Pera J, Reiner AP, Rothwell PM, Sharma P, Slowik A, Sudlow CL, Tatlisumak T, Thijs V, Vicente AM, Woo D, Seshadri S, Saleheen D, Rosand J, Markus HS, Worrall BB, Dichgans M, Group IA, Wellcome Trust Case Control C. Low-frequency and common genetic variation in ischemic stroke: the METASTROKE collaboration. Neurology. 2016;86(13):1217-26.

15. Maebuchi D, Arima H, Doi Y, Ninomiya T, Yonemoto K, Tanizaki Y, Kubo M, Hata J, Matsumura K, lida M, Kiyohara Y. QT interval prolongation and the risks of stroke and coronary heart disease in a general Japanese population: the Hisayama study. Hypertens Res. 2010;33(9):916-21.

16. Veglio M, Giunti S, Stevens LK, Fuller JH, Perin PC, Group EICS. Prevalence of Q-T interval dispersion in type 1 diabetes and its relation with cardiac ischemia: the EURODIAB IDDM Complications Study Group. Diabetes Care. 2002;25(4):702-7.

17. Guo X, Li Z, Guo L, Yu S, Yang H, Zheng L, Pan G, Zhang Y, Sun Y, Pletcher MJ. Effects of metabolically healthy and unhealthy obesity on prolongation of corrected QT interval. Am J Cardiol. 2017:119(8):1199-204.

18. Mohlenkamp S, Schmermund A, Lehmann N, Roggenbuck U, Dragano N, Stang A, Moebus S, Beck EM, Schluter C, Sack S, Meinertz T, Taylor A, Jockel KH, Erbel R, Heinz Nixdorf Recall Study I. Subclinical coronary atherosclerosis and resting ECG abnormalities in an unselected general population. Atherosclerosis. 2008;196(2):786-94.

19. Nielsen JB, Graff C, Pietersen A, Lind B, Struijk JJ, Olesen MS, Haunso S, Gerds TA, Svendsen JH, Kober L, Holst AG. J-shaped association between QTC interval duration and the risk of atrial fibrillation: results from the Copenhagen ECG study. J Am Coll Cardiol. 2013;61(25):2557-64.

20. Yang PS, Pak HN, Park DH, Yoo J, Kim TH, Uhm JS, Kim YD, Nam HS, Joung $\mathrm{B}$, Lee $\mathrm{MH}$, Heo JH. Non-cardioembolic risk factors in atrial fibrillationassociated ischemic stroke. PLoS ONE. 2018;13(7):e0201062.

21. Fauchier L, Maison-Blanche P, Forhan A, D'Hour A, Lepinay P, Tichet J, Vol S, Coumel P, Fauchier JP, Balkau B. Association between heart ratecorrected QT interval and coronary risk factors in 2,894 healthy subjects (the DESIR Study). Data from an epidemiological study on the insulin resistance syndrome. Am J Cardiol. 2000;86(5):557-9.
22. Chugh SS, Reinier K, Singh T, Uy-Evanado A, Socoteanu C, Peters D, Mariani R, Gunson K, Jui J. Determinants of prolonged QT interval and their contribution to sudden death risk in coronary artery disease: the Oregon Sudden Unexpected Death Study. Circulation. 2009;1 19(5):663-70.

23. Katholi RE, Couri DM. Left ventricular hypertrophy: major risk factor in patients with hypertension: update and practical clinical applications. Int J Hypertens. 2011;2011:495349.

24. Park J, Joung B, Uhm JS, Young Shim C, Hwang C, Hyoung Lee M, Pak HN. High left atrial pressures are associated with advanced electroanatomical remodeling of left atrium and independent predictors for clinical recurrence of atrial fibrillation after catheter ablation. Heart Rhythm. 2014;11(6):953-60.

25. Lee JS, Shim CY, Wi J, Joung B, Ha JW, Lee MH, Pak HN. Left ventricular diastolic function is closely associated with mechanical function of the left atrium in patients with paroxysmal atrial fibrillation. Circ J. 2013;77(3):697-704

26. Kang KW, Kim TH, Park J, Uhm JS, Joung B, Hwang C, Lee MH, Pak HN. Long-term changes in heart rate variability after radiofrequency catheter ablation for atrial fibrillation: 1-year follow-up study with irrigation tip catheter. J Cardiovasc Electrophysiol. 2014;25(7):693-700.

27. Oberti C, Wang L, Li L, Dong J, Rao S, Du W, Wang Q. Genome-wide linkage scan identifies a novel genetic locus on chromosome $5 \mathrm{p} 13$ for neonatal atrial fibrillation associated with sudden death and variable cardiomyopathy. Circulation. 2004;110(25):3753-9.

28. Holmes MV, Ala-Korpela M, Smith GD. Mendelian randomization in cardiometabolic disease: challenges in evaluating causality. Nat Rev Cardiol. 2017;14(10):577-90

29. Bennett DA, Holmes MV. Mendelian randomisation in cardiovascular research: an introduction for clinicians. Heart. 2017;103(18):1400-7.

\section{Publisher's Note}

Springer Nature remains neutral with regard to jurisdictional claims in published maps and institutional affiliations.
Ready to submit your research? Choose BMC and benefit from:

- fast, convenient online submission

- thorough peer review by experienced researchers in your field

- rapid publication on acceptance

- support for research data, including large and complex data types

- gold Open Access which fosters wider collaboration and increased citations

- maximum visibility for your research: over $100 \mathrm{M}$ website views per year

At BMC, research is always in progress.

Learn more biomedcentral.com/submissions 